\title{
Insect Egg Size and Shape Evolve With Ecology but Not Developmental Rate
}

\section{Citation}

Church, Samuel H., Seth Donoughe, Bruno A. S. De Medeiros, and Cassandra G. Extavour. 2019. Insect Egg Size and Shape Evolve with Ecology but Not Developmental Rate. Nature 571, no. 7763: 58-62.

\section{Permanent link}

http://nrs.harvard.edu/urn-3:HUL.InstRepos:42241195

\section{Terms of Use}

This article was downloaded from Harvard University's DASH repository, and is made available under the terms and conditions applicable to Other Posted Material, as set forth at http:// nrs.harvard.edu/urn-3:HUL.InstRepos:dash.current.terms-of-use\#LAA

\section{Share Your Story}

The Harvard community has made this article openly available.

Please share how this access benefits you. Submit a story.

Accessibility 


\section{Insect egg size and shape evolve with ecology but not developmental rate}

Samuel H. Church*,1, Seth Donoughe*,1,2, Bruno A. S. de Medeiros 1, Cassandra G. Extavour1,3

${ }_{1}$ Department of Organismic and Evolutionary Biology, Harvard University, Cambridge, MA

02138, United States

2 Current address: Department of Molecular Genetics and Cell Biology, University of Chicago, Chicago, IL 60637, United States

3 Department of Molecular and Cellular Biology, Harvard University, Cambridge, MA 02138 , United States

* These authors contributed equally to the work.

Over the course of evolution, the size of life changes dramatically. These changes are thought to be explained by developmental, morphological, and ecological pressures. To perform phylogenetic tests of these hypotheses, we generated a dataset of more than ten thousand descriptions of insect eggs, and combined these with genetic and life-history datasets. We show that across eight orders of magnitude in egg volume variation, the relationship between size and shape itself evolves, such that predicted global patterns of scaling do not adequately explain egg shape diversity. We show that size is not correlated with developmental rate, and that for many insects, egg size is not correlated with adult body size either. Instead, we find that the evolution of parasitoidism and aquatic oviposition help to explain the size and shape diversification. Our study suggests that where eggs are laid, rather than universal allometric 
constants, underlies size and shape evolution.

Size is a fundamental factor in many biological processes. Size may impact ecological interactions1,2, it scales with features of morphology and physiology3, and larger animals often have higher fitness4. Previous studies have aimed to identify the macroevolutionary forces that explain observed size distributions1, 5, 6, but limited data on the phylogenetic distribution of size has precluded robust tests of these predicted forces4,7. Here we address this problem by assembling a dataset of insect egg phenotypes with sufficient taxon sampling to rigorously test hypotheses about the causes and consequences of size evolution in a phylogenetic framework.

Insect eggs are a compelling system in which to test these macroevolutionary hypotheses. Egg morphologies are extraordinarily diverse, yet they can be readily compared across distant lineages using quantitative traits. Changes in egg size have been studied in relation to changes in other aspects of organismal biology9, including adult body size10-12, features of adult anatomy13, and offspring fitness via maternal investment14. Eggs must also withstand the physiological challenges of being laid in diverse microenvironments, including in water, air, or internal to plants or animals15. Furthermore, because the fertilized egg is the homologous, single-cell stage in the lifecycle of multicellular organisms, egg size diversity is relevant to both cell size and organism size evolution $8,14$.

Three classes of hypotheses have been proposed to explain the evolution of egg size and shape: [1] geometric constraints due to the physical scaling of size and shape13, 16-19, [2] the interaction between size and the rate of development20-22, and [3] the diversification of size and shape response to ecological or life-history changes $10,13,15,23$. We use a phylogenetic approach to test all 
three of these hypotheses, and show that many presumed universal patterns in size, shape, and embryonic development are not supported across insects. Instead, we find that models accounting for ecological change best explain extant egg morphological diversity.

Using custom bioinformatic tools, we assembled a dataset of 10,449 published descriptions of eggs, comprising 6,706 species, 526 families, and every extant hexapod order (Figs. 1a, S1)24. We combined this dataset with backbone hexapod phylogenies25, 26 that we enriched to include taxa in the egg morphology dataset (Fig. S2), and used it to describe the distribution of egg shape and size (Fig. 1b). Our results showed that insect eggs span more than eight orders of magnitude in volume (Figs. 1a, 1c, S3), and revealed new candidates for the smallest and largest described insect eggs: respectively, a parasitoid wasp Platygaster vernalis27 (volume $=7 \times 10-7 \mathrm{~mm} 3$ ), and an earth-boring beetle, Bolboleaus hiaticollis28 (volume $=5$ x $102 \mathrm{~mm} 3)($ Fig. 1c).

Plotting eggs by morphology revealed that some shapes evolved only in certain clades (Fig. 1a, S4-S7). Oblate ellipsoid eggs (aspect ratio <1) are found only in stoneflies, moths and butterflies (Plecoptera, Fig. S4, and Lepidoptera, Fig. 1c image 4, S5). Egg cases (oothecae) have evolved in multiple lineages29. To test whether oothecae constrain shape or size, we measured individual eggs within cases, and found that these are morphologically similar to freely laid relatives (Fig. S8). The most prominent pattern was that distantly related insects have converged on similar morphologies many times independently (Fig. 1a, S7). This high degree of morphological convergence allowed us to robustly test trait associations across independent evolutionary events.

\section{Evolutionary allometry of insect eggs}

Two opposing hypotheses based on predicted geometric constraints have been proposed to explain 
the evolutionary relationship between egg shape and size. Hypothesis A: When eggs evolve to be larger, they get wider (increases in egg size are associated with decreases in aspect ratio)17, 18. This hypothesis predicts a reduction in relative surface area as size increases, which has been proposed as a solution to the presumed cost of making eggshell material18. Hypothesis $B$ : When eggs evolve to be larger, they get longer (increases in egg size are associated with increases in aspect ratio) 13, 18, 19. This hypothesis predicts a reduction in relative cross-sectional area as eggs get larger, which has been proposed as a solution to the need for eggs to pass through a narrow opening during oviposition 13, 19.

To test these hypotheses about the physical scaling of size and shape, we began by modeling the individual evolutionary history of each morphological trait. This allowed us to first determine whether distributions of extant shape and size have been shaped by phylogenetic relationships, or if these traits are instead stochastically distributed. For egg volume, aspect ratio, asymmetry, and angle of curvature (Fig. 1d), we compared four models of evolution: Brownian motion (BM), Brownian motion with evolutionary friction (Ornstein-Uhlenbeck, OU), Brownian motion with a decreasing rate of evolution (Early Burst, EB), and a non-phylogenetic model of stochastic motion (white noise, WN). We found strong evidence that models accounting for phylogenetic covariance fit our data better than a non-phylogenetic model (WN); in other words, insect egg morphology tends to be similar in closely related insects (Table S5). For egg size and aspect ratio, an 'EarlyBurst' (EB) model in which evolutionary rate decreases over time, best describes the observed data. This change in rate is distributed non-uniformly over the insect phylogeny, with some clades evolving faster than others (Figs. S9-S11). In earlier studies EB models were rarely detected30; however our findings are consistent with recent work evaluating datasets that, like ours, comprise 
many taxa and orders of magnitude in morphological variation31, 32. Having established the phylogenetic models that best describe egg trait evolution, we used these results to test hypotheses about the relationship between egg shape and size.

To test which aforementioned scaling relationship best describes insect egg evolution, we compared support for Hypotheses A and B using a phylogenetic generalized least-squares approach (PGLS) to determine the scaling exponent of length and width (the slope of the regression of log-length and log-width). A slope less than one would support Hypothesis A, while a slope greater than one would support Hypothesis B33. An alternative, Hypothesis C, is that egg shape remains the same as size changes, which would result in a slope near one (an isometric relationship; hypotheses shown in Fig. 2a-d). We found that Hypothesis B is best supported across all insects: larger eggs have higher aspect ratios than smaller eggs $(0<$ p-value $<0.005$, slope $=0.78$, Fig. 2 e, Table S6), even when controlling for adult body size (Fig. S14, Table S8). We found no support for Hypothesis A, suggesting that future hypotheses of egg shell evolution may need to account for additional factors such as chorion composition and thickness when considering cost. However, the allometric relationship between size and shape evolves dynamically across the phylogeny, as has also been shown for metabolic scaling in mammals34. Hypothesis $\mathrm{C}$ could not be rejected for beetles and their relatives, nor for butterflies, moths, and caddisflies (respectively Neuropteroidea, p-value of alternative hypothesis test $=0.04$, and Amphiesmenoptera, p-value $=0.01 ;$ Fig. $2 f$, S12, Table S7). Calculating the scaling relationship on lineage subgroups revealed that additional clades, including mayflies, crickets, and shield bugs, also show an isometric relationship (Fig. S13). The marked differences in scaling exponents are evidence that egg evolution was not governed by a universal allometric constant. Instead, evolutionary forces beyond the constraints 
of physical scaling (e.g. development or ecology) are required to explain egg morphological diversification.

\section{Developmental traits and egg evolution}

The egg is the starting material for embryogenesis, and the size of the hatchling is directly related to the size of the egg at fertilization35. It was reported that embryogenesis takes longer in species with larger eggs22, and that this relationship could influence size evolution20, 21. This would be consistent with the observation that larger adult species have lower metabolic rates than smaller species36. To test this prediction across our egg dataset, we assembled published embryological records, and found that indeed, simply comparing egg volume and duration of embryogenesis yields the previously reported positive relationship22 (Fig. S17). However, a linear regression that does not account for phylogenetic relationships is inappropriate for this analysis due to the covariance of traits on an evolutionary tree37. When we accounted for phylogenetic covariance, we found that there is no significant relationship between egg size and duration of embryogenesis across insects, such that eggs of very different sizes develop at a similar rate and vice versa (0.02 $<$ p-value $<0.10$; Fig. 3b, Table S11). These results suggest that the often-invoked trade-off between size and development20-22 does not hold across insects.

We also tested the hypothesis that the size of the egg has a positive relationship with adult body size. Previous work reported this relationship in subsets of insects, and moreover suggested that smaller insects lay proportionally larger eggs for their bodies11,35, 38. Such a relationship between egg size and body size would result in an allometric scaling exponent less than one. We combined 
our dataset of egg size with published adult body length data for insect families39, and found that this relationship was not generalizable across all insect lineages. For example, in flies and their relatives (Antliophora) and mayflies and odonates (Palaeoptera), egg size is not predicted by body size, meaning insects of similar body size lay eggs of different sizes ( $p$-value, Antliophora = 0.02, Palaeoptera = 0.19; Fig. 3c, 3d, Table S12). In Polyneoptera, thrips and true bugs (Condylognatha), and bees, ants, and wasps (Hymenoptera), an isometric relationship between egg size and body size cannot be rejected ( $\mathrm{p}$-value of alternative hypothesis test, Polyneoptera $=0.02$, Hymenoptera $=0.01$, Condylognatha $=0.01$, Fig. S18, Table S13). In general, the predictive power of the relationship between body size and egg size is low: average egg volume can vary by up to four orders of magnitude among species with similar body size (Fig. 3c).

At the time of fertilization an egg is a single cell. Therefore we tested whether the size of this cell evolved with the size of the genome, as has been observed for other cell types40, using a database of genome size for hexapods41. Although the data appeared to show a positive relationship between egg size and genome size (Table S14), we found that this relationship was driven entirely by the lineage Polyneoptera (and specifically grasshoppers, Acrididae). This lineage has evolved genome sizes an order of magnitude larger than other insects and has relatively large eggs (Fig. S19). Across other insect lineages, egg volume and genome size are not significantly related $(0<\mathrm{p}-$ value $<0.08$, Table S14), and egg volume can range over six orders of magnitude for species with similar genome size (Fig. S19c). This indicates that genome size is not a general driver of egg size. The decoupling of genome size, body size, and developmental rate from egg size evolution suggests that insect egg diversification has not been universally constrained by development. 


\section{Oviposition ecology explains egg morphology}

Egg size and shape have been predicted to evolve in response to changes in life-history and ecology. Recent work in birds has highlighted one such relationship, suggesting that birds with increased flight capability have more elliptical and asymmetrical eggs 13. We asked whether an analogous relationship exists between insect flight capability and egg shape. Unlike birds, insects have undergone hundreds of evolutionary shifts to flightless and even wingless forms 42 . We focused on two clades in which flight evolution has been extensively studied. Stick insects (Phasmatodea) have flightless and wingless species43, 44 (Fig. S22), and many butterflies (Lepidoptera) show migratory behavior45, a proxy for increased flight capability relative to nonmigratory taxa (Fig. S22). We found that, in contrast to birds, evolutionary changes in flight ability in these two insect clades were not associated with changes in egg shape (Ornstein-Uhlenbeck model with multiple optima per regime, OUM; $\triangle \mathrm{AICc}<2$, exact values in Tables S18, S19).

Like flight capacity, the microenvironment that insect eggs experience varies widely, including being exposed to air, submerged or floating in water, or contained within a host animals (Fig. 4a). Each microenvironment places different demands on the egg, such as access to oxygen and water during development15. Preliminary studies in small groups of insects suggested that evolutionary changes in oviposition ecology and life history may drive the evolution of egg size and shape $10,23$. To test this prediction across all insects, we compiled records on two oviposition ecology modes that have been extensively studied: oviposition within an animal host (internal parasitic oviposition) and oviposition in or on water. For each mode we reconstructed ancestral changes along the insect phylogeny, and found that both aquatic and internal parasitic oviposition have

been gained and lost multiple times independently (Fig. 4a, 4b, S20, S21). This extensive 
convergent evolution allowed us to perform a strong test of whether egg size and shape evolution are explained by the evolution of oviposition ecology.

We found that the evolution of new oviposition environments is linked to changes in egg size and shape. Models that accounted for shifts to either aquatic or internal parasitic oviposition better explained size and shape distributions than models that did not (OUM, $\triangle \mathrm{AICc}>2$, exact values in Tables S15, S16, S17). In this analysis we compared model fit for each ecology-trait pair separately, and found that these two ecological states were correlated with different egg morphologies. Specifically, shifts to aquatic oviposition were significantly associated with the evolution of smaller eggs with a lower aspect ratio (Fig. 4c, 4d, Table S15), while shifts to internal parasitic oviposition are significantly associated with smaller, more asymmetric eggs (Figs. 4c, 4e, Table S17). Moreover, we note that the smallest eggs are from parasitoid wasps that develop polyembryonically (i.e. multiple embryos form from a single egg 46; Fig. S23). Neither oviposition mode is associated with consistent changes in the allometric relationship between size and shape (Fig. S24).

Given that OU models can be favored when dataset size and measurement error are large 47, we repeated these analyses 100 times using simulated ecological states independent of egg morphological traits. The results of this bootstrap analysis showed that our observed result favoring ecological models of morphological evolution is unlikely to be caused by dataset size alone ( $\mathrm{p}$-value $=0.01$, Table S20). Additionally, these results were robust to uncertainty in phylogenetic relationships and uncertainty in how taxa were classified for oviposition ecology (Tables S16). These findings are evidence that the microenvironment experienced by the egg 
played an important role in morphological evolution.

Insect eggs present an ideal case for testing the predictability of macroevolutionary patterns in size and shape. By comparing insect egg size and shape, we found that previous hypotheses about evolutionary trade-offs with developmental time, body size, or the presumed cost of egg shells do not hold. Although we showed that developmental time is not linked to egg size, we suggest that other features of development (e.g. cell number and distribution) may scale in predictable ways across eight orders of magnitude in egg size. Finally, we provide evidence that the ecology of oviposition drives the evolution of egg size and shape.

1. Peters, R. H. The Ecological Implications of Body Size. Cambridge studies in ecology (Cambridge University Press, Cambridge [Cambridgeshire] ; New York, 1983).

2. Allen, R. M., Buckley, Y. M. \& Marshall, D. J. Offspring size plasticity in response to intraspecific competition: an adaptive maternal effect across life-history stages. The American Naturalist 171, 225-237 (2007).

3. Blanckenhorn, W. U. The evolution of body size: what keeps organisms small? The Quarterly Review of Biology 75, 385-407 (2000).

4. Kingsolver, J. G. \& Pfennig, D. W. Individual-level selection as a cause of Cope's rule of phyletic size increase. Evolution 58, 1608-1612 (2004).

5. Stanley, S. M. An explanation for Cope’s rule. Evolution 27, 1-26 (1973).

6. LaBarbera, M. Analyzing body size as a factor in ecology and evolution. Annual Review of Ecology and Systematics 20, 97-117 (1989). 
7. Chown, S. L. \& Gaston, K. J. Body size variation in insects: a macroecological perspective. Biological Reviews 85, 139-169 (2010).

8. Hinton, H. E. Biology of Insect Eggs, vol. I, II, III (Pergammon Press, Oxford, 1981).

9. Thompson, D. W. On Growth and Form (The University Press; The Macmillan Company, Cambridge, 1942).

10. Fox, C. W. \& Czesak, M. E. Evolutionary ecology of progeny size in arthropods. Annual Review of Entomology 45, 341-369 (2000).

11. Berrigan, D. The allometry of egg size and number in insects. Oikos 60, 313-321 (1991).

12. García-Barros, E. Body size, egg size, and their interspecific relationships with ecological and life history traits in butterflies (Lepidoptera: Papilionoidea, Hesperioidea). Biological Journal of the Linnean Society 70, 251-284 (2000).

13. Stoddard, M. C. et al. Avian egg shape: Form, function, and evolution. Science 356, 12491254 (2017).

14. Bernardo, J. The particular maternal effect of propagule size, especially egg size: patterns, models, quality of evidence and interpretations. American Zoologist 36, 216-236 (1996).

15. Hinton, H. Respiratory systems of insect egg shells. Annual Review of Entomology 14, 343368 (1969).

16. Legay, J. M. Allometry and systematics of insect egg form. Journal of Natural History 11, 493-499 (1977).

17. Blackburn, T. Evidence for a 'fast-slow' continuum of life-history traits among parasitoid 
Hymenoptera. Functional Ecology 65-74 (1991).

18. Kratochvíl, L. \& Frynta, D. Egg shape and size allometry in geckos (Squamata: Gekkota), lizards with contrasting eggshell structure: why lay spherical eggs? Journal of Zoological Systematics and Evolutionary Research 44, 217-222 (2006).

19. Bilder, D. \& Haigo, S. L. Expanding the morphogenetic repertoire: perspectives from the Drosophila egg. Developmental Cell 22, 12-23 (2012).

20. Steele, D. \& Steele, V. Egg size and duration of embryonic development in Crustacea. Internationale Revue der Gesamten Hydrobiologie und Hydrographie 60, 711-715 (1975).

21. Sargent, R. C., Taylor, P. D. \& Gross, M. R. Parental care and the evolution of egg size in fishes. The American Naturalist 129, 32-46 (1987).

22. Maino, J. L. \& Kearney, M. R. Ontogenetic and interspecific metabolic scaling in insects. The American Naturalist 184, 695-701 (2014).

23. Iwata, K. \& Sakagami, S. F. Gigantism and dwarfism in bee eggs in relation to the modes of life, with notes on the number of ovarioles. Japanese Journal of Ecology 16, 4-16 (1966).

24. Church, S. H., Donoughe, S. D., De Medeiros, B. A. S. \& Extavour, C. G. A dataset of egg size and shape from more than 6,700 insect species. Scientific Data (2019). doi:10.1038/s41597019-0049-y

25. Misof, B. et al. Phylogenomics resolves the timing and pattern of insect evolution. Science 346, 763-767 (2014).

26. Rainford, J. L., Hofreiter, M., Nicholson, D. B. \& Mayhew, P. J. Phylogenetic distribution of extant richness suggests metamorphosis is a key innovation driving diversification in insects. 
PLoS One 9, 1-7 (2014).

27. Leiby, R. \& Hill, C. The polyembryonic development of Platygaster vernalis. Journal of Agricultural Research 28, 829-839 (1924).

28. Houston, T. F. Brood cells, life-cycle stages and development of some earth-borer beetles in the genera Bolborhachium, Blackburnium and Bolboleaus (Coleoptera: Geotrupidae), with notes on captive rearing and a discussion of larval diet. Austral Entomology 55, 49-62 (2016).

29. Goldberg, J. et al. Extreme convergence in egg-laying strategy across insect orders. Scientific Reports 5, 7825 (2015).

30. Harmon, L. J. et al. Early bursts of body size and shape evolution are rare in comparative data. Evolution 64, 2385-2396 (2010).

31. Uyeda, J. C., Hansen, T. F., Arnold, S. J. \& Pienaar, J. The million-year wait for macroevolutionary bursts. Proceedings of the National Academy of Sciences of the United States of America 108, 15908-15913 (2011).

32. Cooper, N. \& Purvis, A. Body size evolution in mammals: complexity in tempo and mode. The American Naturalist 175, 727-738 (2010).

33. Peters, R. H. \& Wassenberg, K. The effect of body size on animal abundance. Oecologia $\mathbf{6 0}$, 89-96 (1983).

34. Sieg, A. E. et al. Mammalian metabolic allometry: do intraspecific variation, phylogeny, and regression models matter? The American Naturalist 174, 720-733 (2009).

35. Polilov, A. A. Small is beautiful: features of the smallest insects and limits to miniaturization. Annual Review of Entomology 60, 103-121 (2015). 
36. Gillooly, J. F., Brown, J. H., West, G. B., Savage, V. M. \& Charnov, E. L. Effects of size and temperature on metabolic rate. Science 293, 2248-2251 (2001).

37. Felsenstein, J. Phylogenies and the comparative method. The American Naturalist 125, 115 (1985).

38. Rensch, B. Histological changes correlated with evolutionary changes of body size. Evolution 2, 218-230 (1948).

39. Rainford, J. L., Hofreiter, M. \& Mayhew, P. J. Phylogenetic analyses suggest that diversification and body size evolution are independent in insects. BMC Evolutionary Biology 16, 8 (2016).

40. Gregory, T. R. Coincidence, coevolution, or causation? DNA content, cell size, and the Cvalue enigma. Biological Reviews 76, 65-101 (2001).

41. Gregory, T. R. Animal Genome Size Database (2019). URL http://www.genomesize. com.

42. Roff, D. A. The evolution of flightlessness in insects. Ecological Monographs 60, 389-421 (1990).

43. Whiting, M. F., Bradler, S. \& Maxwell, T. Loss and recovery of wings in stick insects. Nature 421, 264 (2003).

44. Trueman, J., Pfeil, B., Kelchner, S. \& Yeates, D. Did stick insects really regain their wings? Systematic Entomology 29, 138-139 (2004).

45. Stancă-Moise, C. et al. Migratory species of butterflies in the surroundings of Sibiu (Romania). Scientific Papers Series Management, Economic Engineering in Agriculture and Rural Development 16, 319-324 (2016). 
46. Ivanova-Kasas, O. M. Polyembryony in insects. In Developmental Systems: Insects, 243271 (Academic Press, London, 1972).

47. Cooper, N., Thomas, G. H., Venditti, C., Meade, A. \& Freckleton, R. P. A cautionary note on the use of Ornstein Uhlenbeck models in macroevolutionary studies. Biological Journal of the Linnean Society 118, 64-77 (2016).

48. Nieves-Uribe, S., Flores-Gallardo, A., Hernández-Mejía, B. C. \& Llorente-Bousquets, J. Exploración morfológica del corion en Biblidinae (Lepidoptera: Nymphalidae): aspectos filogenéticos y clasificatorios. Southwestern Entomologist 40, 589-648 (2015).

49. Barata, J. M. S. Morphological aspects of Triatominae eggs: II. macroscopic and exochorial characteristics of ten species of the genus Rhodnius Stal, 1859 (Hemiptera-Reduviidae). Revista de Saúde Pública 15, 490-542 (1981).

50. Iwata, K. The comparative anatomy of the ovary in Hymenoptera. (records on 64 species of Aculeata in Thailand, with descriptions of ovarian eggs). Mushi 38, 101-109 (1965).

51. Dutra, V. S., Ronchi-Teles, B., Steck, G. J. \& Silva, J. G. Egg morphology of Anastrepha spp. (Diptera: Tephritidae) in the fraterculus group using scanning electron microscopy. Annals of the Entomological Society of America 104, 16-24 (2011). 
Supplementary Information Supplementary Information is linked to the online version of the paper at www.nature.com/nature.

Acknowledgements This work was supported by the National Science Foundation (NSF) under Grant No. IOS-1257217 to CGE, and NSF Graduate Research Training Fellowship No. DGE1745303 to SHC, and by a Jorge Paulo Lemann Fellowship to BdM from Harvard University. We thank the Extavour lab and Brian Farrell for discussion, and Casey Dunn, Dakota McCoy, Dan Rice, Albert Kao, Elena Kramer, Jack Boyle, Leonora Bittleston, Mansi Srivastava, Milo Johnson, Peter Wilton, Richard Childers, and Sofia Prado-Irwin for suggestions on initial versions of this manuscript. We acknowledge the Ernst Mayr Library at the Museum of Comparative Zoology at Harvard, and specifically Mary Sears, for assistance in gathering the references used in this study.

Author contributions SHC and SD contributed equally to dataset generation, experimental design, data analysis, writing, and figure preparation. SHC wrote all code to perform statistical analyses. BdM performed phylogenetic analyses. BdM and CGE contributed to experimental design, interpretation, and writing.

Author information Reprints and permissions information is available at www.nature.com/reprints. The authors declare no competing interests. Correspondence and requests for materials should be addressed to SHC (church@g.harvard.edu) and CGE (extavour@oeb.harvard.edu).

\section{Figure Legends}


1. The shapes and sizes of hexapod eggs. a, Eggs plotted in a morphospace defined by volume (mm3) and aspect ratio (unitless), log scale. Points are colored by clades in $\mathbf{b}$, with relationships according to Misof et al. 201425, one of the backbone phylogenies used in this study. Numbered points correspond to six eggs in $\mathbf{c}$, selected to show a range of sizes and shapes, arranged by aspect ratio27, 28, 48-51. d, Size and shape are described using six features, calculated as shown.

\section{The allometric relationship of egg shape and size evolves across insects.} Hypothesized relationships between size and shape: a, larger eggs are proportionally wider, (solid line); b, larger eggs are proportionally longer (dotted line); c, shape and size scale isometrically (dashed line). d, Each hypothesis predicts a different scaling exponent - the slope of the regression between log-length and log-width. e, Egg length and width plotted in log-log space. Dashed line represents a hypothetical 1:1 relationship (c). Solid lines are clade-specific PGLS regressions; points are randomly selected representatives per genus. $\mathrm{n}=$ genera: Palaeoptera $=104$, Polyneoptera $=262$, Condylognatha $=202$, Hymenoptera $=356$, Neuropteroidea $=265$, Amphiesmenoptera $=76$, Antliophora $=199 . \mathbf{f}$, The distribution of scaling exponents from PGLS regressions of seven monophyletic clades, calculated over the posterior distribution. White lines, boxes, bars, and dots represent median, 25-to-75th percentiles, 5-to-95th percentiles, and outliers. Asterisks indicate a significant relationship ( $\mathrm{p}$-value $<0.01$, exact values in Table S6), and doubledaggers indicate the relationship is not distinguishable from isometry (p-value $>0.01$, exact values in Table S7). $\mathrm{n}=100$ PGLS regressions. Colors correspond to Fig. $1 \mathrm{~b}$.

3. Developmental features do not co-vary with egg size. a, Mature eggs undergo embryonic development, hatch, and grow into adults. b, Egg volume (mm3) compared to duration 
of embryogenesis, defined as time from egg-laying to hatching (hours), adjusted for incubation temperature. When phylogeny is accounted for, there is no significant relationship. c, Egg volume (mm3) compared to adult body volume, calculated as body length cubed (mm3). Dashed line represents a hypothetical 1:1 relationship (isometry). Solid lines are clade-specific PGLS phylogenetic regressions; points are family- or order-level average egg size and median adult size. $\mathrm{n}=$ family- or order-level averages: Palaeoptera $=15$, Polyneoptera $=31$, Condylognatha $=36$, Hymenoptera $=44$, Neuropteroidea $=36$, Amphiesmenoptera $=31$, Antliophora $=39$. d, The distribution of scaling exponents from PGLS regressions of seven monophyletic clades. White lines, boxes, bars, and dots represent median, 25-to-75th percentiles, 5-to-95th percentiles, and outliers. Asterisks indicate a significant relationship (p-value $<0.01$, exact values in Table S12), and double-daggers indicate the relationship is not distinguishable from isometry (p-value $>0.01$, exact values in Table S13). $\mathrm{n}=100$ PGLS regressions. Colors correspond to Fig. 1b.

\section{Shifts in oviposition ecology are associated with changes in egg morphology. a, Two}

modes of oviposition ecology: laying eggs within an animal host (orange; e.g. parasitoid wasps), and in water (blue; e.g. mosquitoes). Other oviposition substrates (e.g. terrestrial, within plants) shown in gray. b, Ancestral state reconstruction of oviposition mode reveals both evolved multiple times (see Fig. S17, S18). c-f, The distribution of egg features, colored by ecology: c, volume

(mm3, log scale); d, aspect ratio (unitless, log scale); e, asymmetry (unitless); and $\mathbf{f}$, angle of curvature (degrees). Asterisks indicate that the model accounting for ecology fits the data better (OUM, $\triangle \mathrm{AICc}>2$, exact values in Table S14-S19) than a non-ecological model.

\section{Methods}


Data availability The dataset of insect eggs is publicly available at Dryad https://datadryad. org/review?doi=doi:10.5061/dryad.pv40d2r and described in Church et al. $2019_{24}$.

The phylogenetic posterior distributions are provided as supplemental files 'phylogeny_posterior

distribution_misof_backbone.nxs' and 'phylogeny_posterior_distribution_rainford_backbone.nxs'.

Code availability All code required to reproduce the analyses and figures shown here is available at https://github.com/shchurch/Insect_Egg_Evolution.

Creating the insect egg dataset $\mathrm{A}$ list of the 1,756 literature sources used to generate the egg dataset is provided as a supplemental file, 'bibliography egg dataset.pdf'. A full description of the methods used to assemble the insect egg dataset have been published separately24. Egg descriptions were collected from published accounts of insect eggs using custom software to parse text from PDFs and measure published images (Fig. 1b), followed by manual verification. Each entry in the egg dataset includes a reference to an insect genus and, when reported, species name. Scientific names were validated using TaxReformer24, which relies on online taxonomic databases52-56.

Measuring egg features Full trait definitions are described in the Supplementary Information and summarized briefly below. To resolve ambiguous cases and to measure published images, we used the definitions below.

Egg length: We defined egg length as the distance in millimeters $(\mathrm{mm})$ of the axis of rotational symmetry.

Egg width: We defined egg width as the widest diameter (mm), measured perpendicular to the axis of rotational symmetry of the egg. For eggs described in published records as having both a width 
and breadth or depth (i.e. the egg is a flattened ellipsoid57), we defined width as the wider of the two diameters, and breadth as the diameter perpendicular to both the width and length.

Egg volume: Volume (mm3) was calculated using the equation for the volume of an ellipsoid: $1 / 6 \pi \mathrm{lw} 2$, following previous workers12, 58 .

Egg aspect ratio: Aspect ratio was calculated as the ratio of length to width.

Egg asymmetry: Asymmetry was calculated as the ratio between the two egg diameters at the first and third quartile of the length axis, minus one. The first quartile was always defined as the larger of the two diameters.

Angle of egg curvature: The angle of curvature was measured as the angle (degrees) of the arc created by the endpoints and midpoint of the length axis.

Phylogenetic methods A genus-level phylogeny was built by combining mitochondrial 18S and 28S sequence data from the SILVA database59-62 with phylogenetic constraints from published higher-level insect phylogenies25, 26. To account for phylogenetic uncertainty in comparative analyses, trees were estimated using a hierarchical approach63, 64. Separate phylogenies for each insect order were inferred in a Bayesian framework using MrBayes v. 3.2.665 and 100 post-burnin trees were randomly chosen for each order using the order-level backbone trees of Rainford et al. 201426 and Misof et al. 201425. See Supplementary Information, section "Sequence alignment and phylogenetic methods" for more details.

Annotating the egg dataset with developmental trait data For developmental traits, a set of references were assembled from the embryological and ecological literature, and then used to compile data on interval between syncytial mitoses, time to cellularization, and duration of 
embryogenesis. Developmental rate observations were rescaled to approximate rates at a standardized temperature of $20^{\circ} \mathrm{C}$ following previous work66. For a full list of sources, methods used in this calculation, and further discussion of developmental trait definitions, see the Supplementary Information, section "Collecting developmental time data".

Annotating the egg dataset with life-history trait data For each of the ecological features of interest (internal parasitic oviposition, aquatic oviposition, flightlessness, and migratory behavior) taxonomic descriptions from the literature were matched to taxa in the insect egg dataset. For some taxonomic groups it was not possible to classify all members unambiguously. In these cases, the ecological state was coded "uncertain", and the potential impact of this uncertainty on results was tested. For each trait the ancestral state reconstruction was estimated using an equal-rates model (R package corHMM67, function rayDISC, node.states='marginal'). For a full list of sources and methods used see the Supplementary Information, section "Evolutionary history of ecological traits".

\section{Data analysis and evolutionary model comparison}

Egg length, width, volume, and aspect ratio were $\log _{10}$ transformed. Angle of curvature and asymmetry were square root transformed.

Models of evolution were compared using the R package geiger68. For each trait (egg length, width, volume, aspect ratio, asymmetry, and angle of curvature), the model fit of a Brownian Motion (BM), Ornstein-Uhlenbeck (OU), and Early-Burst (EB) model was compared against a null hypothesis of a white noise (WN) model that assumes no evolutionary correlation. See Supplementary Information, section 'Evolutionary model fitting' for details. The performance of the best fitting model was further analyzed by comparing expected values of parameters from 
simulations under the model to observed parameters, using the R package arbutus69.

The ancestral state of volume, aspect ratio, and angle of curvature were mapped on the summary phylogeny using the R package phytools70 (version 0.6-44, function contMap). Evolutionary rate regimes of volume, aspect ratio, and the angle of curvature were fit on the summary phylogeny using the program BAMM71, 72 (version 2.5.0, R package BAMMtools version 2.1.6, setBAMMpriors, prior for expected number of shifts set to 10 , for $10,000,000$ generations).

All evolutionary regression analyses were performed using a phylogenetic generalized least squares (PGLS) approach in the $\mathrm{R}$ packages ape73 (version 5.0, correlation structure = corBrownian) and nlme74 (version 3.1-131.1). Given that the EB models best fit the data, we also tested a corBlomberg correlation structure, which invokes an accelerating-decelerating model of evolution, with the decelerating rate of trait change fixed at 1.3.

For comparisons performed at the genus level, each regression was repeated over 100 trees randomly drawn from the posterior distribution randomly selecting a representative egg dataset entry per genus. For comparisons performed at the family level, each regression was repeated 100 times calculating the family level average egg data from $50 \%$ of entries per family.

For phylogenetic regressions controlling for a third variable, we calculated the phylogenetic residuals of each variable against the dependent variable, and then calculated the phylogenetic regression of the residuals75. To test alternative hypotheses, new data were simulated using a fixed scaling exponent and the parameters of the best fitting model with the $\mathrm{R}$ package phylolm 76 (version 2.5, function 'rTrait').

Allometric regressions were performed over all insect taxa as well as for seven monophyletic 
groups of insects individually (Palaeoptera, Polyneoptera, Condylognatha, Hymenoptera, Neuropteroidea, Amphiesmenoptera, Antliophora). In addition, the scaling exponent between egg length and width was calculated for each monophyletic group of taxa with more than 20 tips but fewer than 50 .

Following ancestral state reconstruction of ecological regimes, for each ecology-trait pair (internal parasitic or aquatic oviposition combined with volume, aspect ratio, asymmetry, or curvature) the fit of a Brownian-Motion model (BM), an Ornstein-Uhlenbeck model with a single optimum (OU1), and an Ornstein-Uhlenbeck model with an independent optimum for each ecological state (OUM) were compared using the R package OUwien7 (version 1.50). These analyses were repeated over 100 trees randomly drawn from the posterior distribution, and randomly selecting a representative egg for each genus.

Plots were generated in R. Figures were assembled with Adobe Illustrator. Egg images that were reproduced from other publications were converted to greyscale, contrast adjusted, rotated, then and masked from their backgrounds using Adobe Photoshop.

Statistical Information For evolutionary regressions and parametric bootstraps, a significance threshold of 0.01 was used. All p-values were rounded to the nearest hundredth. Exact values for all statistical comparisons are available in the figure legends and Supplementary Information. For evolutionary model comparisons, weighted AICc values were compared at a significance threshold of 2. Evolutionary regressions were performed 100 times each, taking into account phylogenetic and phenotypic uncertainty. For more details see Supplementary Information, section "Calculating allometric exponents using phylogenetic generalized least squares (PGLS)”.

52. Patterson, D., Mozzherin, D., Shorthouse, D. P. \& Thessen, A. Challenges with using 
names to link digital biodiversity information. Biodiversity Data Journal (2016).

53. Pyle, R. L. Towards a global names architecture: The future of indexing scientific names. ZooKeys 550, 261-281 (2016).

54. Rees, J. \& Cranston, K. Automated assembly of a reference taxonomy for phylogenetic data synthesis. Biodiversity Data Journal 5, e12581 (2017).

55. Hinchliff, C. E. et al. Synthesis of phylogeny and taxonomy into a comprehensive tree of life. Proceedings of the National Academy of Sciences of the United States of America 112, 12764 12769 (2015).

56. GBIF. GBIF: The Global Biodiversity Information Facility (2018).

57. Clark, J. The capitulum of phasmid eggs (Insecta: Phasmida). Zoological Journal of the Linnean Society 59, 365-375 (1976).

58. Markow, T., Beall, S. \& Matzkin, L. Egg size, embryonic development time and ovoviviparity in Drosophila species. Journal of Evolutionary Biology 22, 430-434 (2009).

59. Glöckner, F. O. et al. 25 years of serving the community with ribosomal RNA gene reference databases and tools. Journal of Biotechnology 261, 169-176 (2017).

60. Quast, C. et al. The SILVA ribosomal RNA gene database project: Improved data processing and web-based tools. Nucleic Acids Research 41, 590-596 (2013).

61. Yilmaz, P. et al. The SILVA and "all-species Living Tree Project (LTP)" taxonomic frameworks. Nucleic Acids Research 42, 643-648 (2014).

62. Pruesse, E., Peplies, J. \& Glöckner, F. O. SINA: Accurate high-throughput multiple sequence 
alignment of ribosomal RNA genes. Bioinformatics 28, 1823-1829 (2012).

63. Smith, S. A. \& Brown, J. W. Constructing a broadly inclusive seed plant phylogeny. American Journal of Botany 105, 302-314 (2017).

64. Jetz, W., Thomas, G. H., Joy, J. B., Hartmann, K. \& Mooers, A. O. The global diversity of birds in space and time. Nature 491, 444-448 (2012).

65. Ronquist, F. et al. MrBayes 3.2: Efficient Bayesian phylogenetic inference and model choice across a large model space. Systematic Biology 61, 539-542 (2012).

66. Maino, J. L., Pirtle, E. I. \& Kearney, M. R. The effect of egg size on hatch time and metabolic rate: theoretical and empirical insights on developing insect embryos. Functional Ecology 31, 227234 (2017).

67. Beaulieu, J. M., O’Meara, B. C. \& Donoghue, M. J. Identifying hidden rate changes in the evolution of a binary morphological character: the evolution of plant habit in campanulid angiosperms. Systematic Biology 62, 725-737 (2013).

68. Harmon, L. J., Weir, J. T., Brock, C. D., Glor, R. E. \& Challenger, W. Geiger: investigating evolutionary radiations. Bioinformatics 24, 129-131 (2007).

69. Pennell, M. W., FitzJohn, R. G., Cornwell, W. K. \& Harmon, L. J. Model adequacy and the macroevolution of angiosperm functional traits. The American Naturalist 186, E33-E50 (2015).

70. Revell, L. J. phytools: an R package for phylogenetic comparative biology (and other things). Methods in Ecology and Evolution 3, 217-223 (2012).

71. Rabosky, D. L. Automatic detection of key innovations, rate shifts, and diversity-dependence on phylogenetic trees. PLoS One 9, e89543 (2014). 
72. Rabosky, D. L. et al. Bamm tools: an R package for the analysis of evolutionary dynamics on phylogenetic trees. Methods in Ecology and Evolution 5, 701-707 (2014).

73. Paradis, E., Claude, J. \& Strimmer, K. APE: analyses of phylogenetics and evolution in R language. Bioinformatics 20, 289-290 (2004).

74. Pinheiro, J., Bates, D., DebRoy, S. \& Sarkar, D. R core team (2014) nlme: linear and nonlinear mixed effects models. $\mathrm{R}$ package version 3.1-117. Available at http://CRAN.Rproject.org/package=nlme (2014).

75. Revell, L. J. Phylogenetic signal and linear regression on species data. Methods in Ecology and Evolution 1, 319-329 (2010).

76. Tung Ho, L. S. \& Ané, C. A linear-time algorithm for Gaussian and non-Gaussian trait evolution models. Systematic Biology 63, 397-408 (2014).

77. Beaulieu, J. M., Jhwueng, D.-C., Boettiger, C. \& O’Meara, B. C. Modeling stabilizing selection: expanding the Ornstein-Uhlenbeck model of adaptive evolution. Evolution 66, 23692383 (2012). 